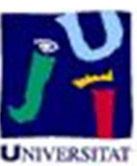

Título artículo / Títol article: Daily Solar Energy Estimation for Minimizing Energy Storage Requirements in PV Power Plants

Autores / Autors

H. Beltrán, E. Pérez, N. Aparicio, P. Rodríguez

Revista:

Sustainable Energy, IEEE Transactions on

Versión / Versió:

Versió post-print

Cita bibliográfica / Cita bibliogràfica (ISO 690):
BELTRÁN, Hector, et al. Daily solar energy estimation for minimizing energy storage requirements in $\mathrm{PV}$ power plants. Sustainable Energy, IEEE Transactions on, 2013, vol. 4, no 2, p. 474481.

url Repositori UJI: 


\title{
Daily Solar Energy Estimation for Minimizing Energy Storage Requirements in PV Power Plants
}

\author{
Hector Beltran, Emilio Pérez, Néstor Aparicio, Member, IEEE, and Pedro Rodríguez, Senior Member, IEEE
}

\begin{abstract}
This paper proposes an optimized energy management strategy (EMS) for photovoltaic (PV) power plants with energy storage (ES) based on the estimation of the daily solar energy production. This EMS produces a constant-by-hours power reference which mitigates the stochastic nature of $P V$ production typically associated to the solar resource, and enables PV power plants to take part in the day and intraday electricity markets. The possibility of using the intraday market sessions to refine the plant's power reference paves the way to minimizing the energy capacity ratings of the ES system required to operate the PV power plant without incurring excessive production deviations. This proposal is analyzed on an annual basis using actual irradiance data and theoretical irradiance models extracted from official databases.
\end{abstract}

Index Terms-Energy storage (ES), photovoltaic (PV) systems, power generation planning.

\section{INTRODUCTION}

$\mathbf{R}$ ENEWABLE energy sources (RES) are growing quickly all over the world thanks to both environmental and geopolitical concerns [1]. However, the intermittent and stochastic production obtained from some of these RES poses technical and economic challenges when integrated on a large scale due to the introduction of significant uncertainties into the operation and planning of the power systems [2]. This is especially important for wind or photovoltaic (PV) power technologies [3]-[5]. Wind power is currently more widespread, globally speaking, and the aforementioned problem has thus already been tackled in countries such as Denmark. Although a traditional solution to the intermittency of wind generation has been primarily based on improving grid interconnection [6], as wind penetration becomes more and more important new solutions have had to be proposed. The intermittency of PV power, on the other hand, is even higher, since it depends on both the day-night cycles and the real-time weather conditions

Manuscript received May 20, 2011; revised April 09, 2012; accepted June 16,2012 . This work was supported in part by the Spanish Ministry of Science and Innovation under the project ENE 2008-06841-C02/ALT, and in part by the Universitat Jaume I de Castelló under Grant P1.1A2008-11.

H. Beltran, E. Pérez, and N. Aparicio are with the Area of Electrical Engineering, Universitat Jaume I, 12071 Castelló de la Plana, Spain (e-mail: hbeltran@uji.es).

P. Rodríguez is with the Electrical Engineering Department, Universitat Politècnica de Catalunya-Barcelona Tech, 08222, Terrassa, Spain (e-mail: prodriguez@ee.upc.edu).

Color versions of one or more of the figures in this paper are available online at http://ieeexplore.ieee.org.

Digital Object Identifier 10.1109/TSTE.2012.2206413 (presence of clouds). Conversely, PV power still represents a low capacity percentage of the electricity production share but current worldwide installing trends [7] indicate that different countries will also face integration problems in a near future.

Energy storage (ES) is identified as one of the potential solutions to deal with this issue [8]-[10]. This is reflected in many studies [4], [11], [12] and demonstration projects, like the one started in 2010 within the Eurogia ${ }^{+}$cluster [13]. Thus, the main benefits of an energy storage system (ESS) in a PV plant is indeed its capability to increase the economic income by enabling these power plants to access the pool electricity market, which, depending on the country and legislation, will present different configurations (with power production scheduling in hourly, or even shorter, periods). The benefits are not limited to the PV plant itself, however, but also extend to the whole power system. The reduction in the instantaneous power fluctuations experienced by a part of the production mix generators thanks to the introduction of ES would make the generation-demand balance easier to accomplish and more stable. This would make it possible to lower the number of units in hot standby (as primary and secondary reserve generators), thus reducing the operation costs of the system while also allowing a greater degree of penetration of RES with power productions that are less intermittent.

This paper presents an energy management strategy (EMS) designed to control a PV power plant, allowing it to generate a constant-by-hours power, thanks to the ES introduction, which enables its participation in the electricity markets. This EMS bases the PV + ES constant production reference on theoretical irradiance models extracted from a public database [14], and refines it throughout the day using a daily solar energy estimation to take advantage of the different intraday electricity market sessions. In this way, it minimizes the ESS capacity ratings required to operate the plant without incurring excessive production deviations and, then, avoiding penalties. The proposal is analyzed on an annual basis using actual irradiance data sampled every $2 \mathrm{~min}$. This annual analysis allows some recommended values to be established with regard to the ESS capacity required by this EMS.

The paper starts by describing the configuration and functioning of the PV power plant with ES. Then, the EMS proposed to qualify this type of power plant with an optimized constant power production is described. This EMS paves the way for them to take part in electricity markets. After that, the daily solar energy estimation mechanism used to adjust the PV + ES power reference on the different sessions of the intraday electricity market is presented in Section III. Section IV is devoted to the calculation of the ESS energy capacities required to operate the PV power plant under the proposed EMS with and without 


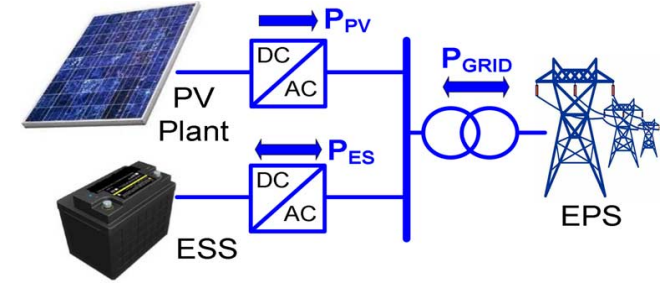

Fig. 1. Schema of the PV + ES power plant topology.

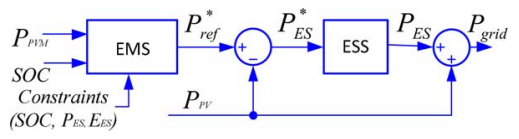

Fig. 2. Block diagram for the control structure of the PV + ES power plant.

intraday adjustments, highlighting the improvements achieved thanks to the daily solar energy estimation mechanism. Finally, some concluding remarks are presented in Section V.

\section{Power Plant Configuration And Control}

Large-scale grid-tied PV power plants could achieve high degrees of penetration and improved performances, thus avoiding much of their intermittent and uncontrollable nature, thanks to the integration of ES units. A simplified schema of a PV + ES power plant configuration is represented in Fig. 1.

The main goal with such a configuration is to be able to release the PV production from its current real-time weather dependence and enable future PV power plants to provide a controlled constant production, which could be traded on electricity markets.

Therefore, since the solar resource presents a highly stochastic behavior, the PV instantaneous production should be completed at each moment by the power exchange performed by the ESS in order to achieve the instantaneous value of power that the power plant has committed with the market. Thus, the ESS operation is managed by the equation

$$
P_{\mathrm{ES}}(t)=\left(P_{\text {ref }}^{*}(t)-P_{\mathrm{pv}}(t)\right)
$$

with $P_{\text {ref }}^{*}$ being the power reference to be submitted by the plant, $P_{\mathrm{PV}}$ the instantaneous power provided by the PV panels, and $P_{\mathrm{ES}}$ the current power exchanged by the ES to compensate for the $\mathrm{PV}$ production. $P_{\mathrm{ES}}$ is, in turns

$$
\begin{aligned}
\text { Discharge }: P_{\mathrm{ES}}>0 \rightarrow d E_{\mathrm{ES}}(t) / d t=-P_{\mathrm{ES}} / \varepsilon_{d} \\
\text { Charge }: P_{\mathrm{ES}}<0 \rightarrow d E_{\mathrm{ES}}(t) / d t=-P_{\mathrm{ES}} \cdot \varepsilon_{c}
\end{aligned}
$$

with $E_{\mathrm{ES}}$ being the stored energy (available energy), $\varepsilon_{c}$ the charging efficiency, and $\varepsilon_{d}$ the discharging efficiency. The $E_{\mathrm{ES}}$ is permanently accounted for by controlling the state-of-charge (SOC) of the ESS. Equation (1) is defined according to the control structure of the plant, represented in Fig. 2. This block diagram describes how the $P_{\text {ref }}^{*}$ is tracked by the combination of the $P_{\mathrm{PV}}$ plus the $P_{\mathrm{ES}}$, thus forming the power supplied to the grid by the whole plant $P_{\text {grid }}$.

Apart from the $P_{\mathrm{PV}}$, which mainly depends on the current solar irradiance, and the $P_{\mathrm{ES}}$, which is calculated by the system, the target power or power commitment to be supplied by the PV power plant, $P_{\text {ref }}^{*}$, is defined by taking into account different constraints and parameters such as the SOC evolution and the PV production model, $P_{\mathrm{PVM}}$ in Fig. 2.

The $P_{\text {ref }}^{*}$ is a key parameter for any power generator to enter the daily electricity market, since it represents the power plant's commitment with the grid operator. Therefore, likely and dispatchable production forecasts are required throughout the daytime to be able to take part in the electricity pool while avoiding penalties deriving from unexpected production deviations. This tradable production is normally defined according to the EMS of the plant. Various EMS for driving PV plants with ES have recently been analyzed in the literature [11], [12], [15]-[17]. A new EMS is proposed here to settle this $P_{\text {ref }}^{*}$ in PV + ES power plants, and it stands out for being specially designed to allow the participation of large-scale grid-tied PV power plants in the electricity market with the introduction of a minimized ESS.

The EMS proposal, called hourly constant-power steps (HCPS) EMS, is based on defining a constant-by-hours $P_{\text {ref }}^{*}$. In order to define the power value for each of the 24 hourly periods, the EMS performs an optimization of the reference using quadratic programming $(\mathrm{QP})$, which solves the problem of optimizing a quadratic function with several variables subject to linear constraints on these variables. The target for the mathematical optimization of $P_{\text {ref }}^{*}$ using QP is to keep the SOC of the ESS as close as possible to a reference value at each moment, in order to require the least ESS energy capacity. This optimization is defined by

$$
\min \sum_{0}^{n}\left(\mathrm{SOC}_{i}-\mathrm{SOC}_{\mathrm{ref}}\right)^{2}
$$

with $\mathrm{SOC}_{i}$ being the ESS charge level at any sampled moment during the analysis, and $\mathrm{SOC}_{\mathrm{ref}}$ the reference $\mathrm{SOC}$ value, which is typically $50 \%$ of the ESS energy capacity - a value that is intended to prevent ESS energy saturations. The total number of samples considered in the optimization is $n=720$, which corresponds to the number of 2-min periods in one day (note that this is the sampling period for the analysis performed in the present paper). This quadratic optimization is subject to the following constraints:

$$
\begin{aligned}
P_{\mathrm{ref}}(t) & = \begin{cases}p_{1}, & t=0 \ldots 30 \\
p_{2}, & t=31 \ldots 60 \\
\ldots & \\
p_{24}, & t=691 \ldots 720\end{cases} \\
P_{\mathrm{ref}}(t) & =P_{\mathrm{pv}}(t)+P_{\mathrm{ES}}(t) \quad t=0, \ldots, 720 \\
P_{\mathrm{min}} & <P_{\mathrm{ES}}(t)<P_{\max } \quad t=0, \ldots, 720 \\
E_{\mathrm{ES}}(t) & =E_{\mathrm{ES}}(t-1)-T \cdot P_{\mathrm{ES}} 0, \ldots, 720 \\
E_{\mathrm{min}} & <E_{\mathrm{ES}}(t)<E_{\max } t=0, \ldots, 720 .
\end{aligned}
$$

Therefore, each of the 24 step values defined with this optimization lasts 30 samples (one hour), as can be observed in (5). Four additional restrictions are introduced. Those in (6) and (7) establish the functioning of the power plant, as already introduced by (1), and some power limitations in the ESS power setpoint (derived from the ESS connection power converter ratings), respectively. On the other hand, restrictions in (8) and (9) are focused on how the SOC of the ESS evolves in the daytime and what the SOC level limits to these variations are. Finally, 


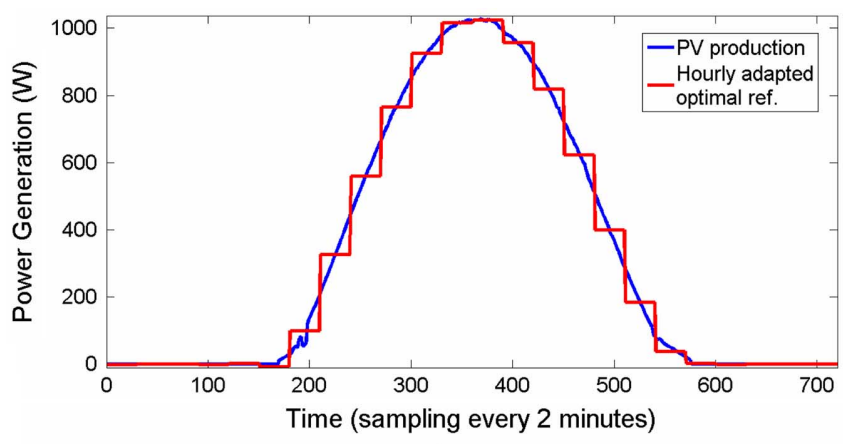

a)

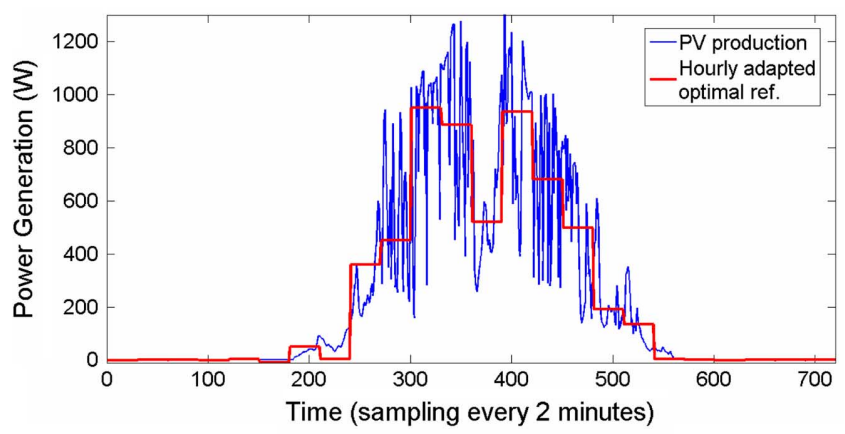

b)

Fig. 3. Ideal $P_{\text {ref }}^{*}$ under the HCPS EMS for (a) a clear and (b) a cloudy day.

note also that neither charging nor discharging efficiency coefficients, which would interfere in (8), have been introduced into the model, since these involve a nonlinear behavior of the system. Nonetheless, these efficiencies have been included in the annually based simulation presented later and performed to determine ESS ratings, in accordance with (2) and (3).

To enter the daily electricity market, the QP calculation will initially be launched the day before the day-on-schedule, thereby providing the 24 -h production program $\left(P_{\text {ref }}^{*}\right)$ that the PV plant operator expects it should be able to generate. It seems essential, then, to use a precise $P_{\mathrm{PVM}}$ in order to obtain an accurate production program. If the exact daily instantaneous $P_{\mathrm{PV}}$ were perfectly well known in advance, perfect $P_{\text {ref }}^{*}$ fits with the actual $P_{\mathrm{PV}}$ would be obtained. This is observed in Fig. 3, where the resulting $P_{\text {ref }}^{*}$ that the QP would ideally fix ( $a$ posteriori) for a clear and a cloudy day are represented.

However, the exact irradiance and, hence, a precise PV production forecast for the day-on-schedule are not available in the moment the $P_{\text {ref }}^{*}$ is initially defined. Therefore, only a rough power commitment based on statistical PV production data and on weather forecasts updated the day before the day-on-schedule can be obtained. Thus, apart from accurate meteorological information sources, solar resource databases such as the European Union PVGIS database are needed [14]. This database is normally utilized for PV power plant long-term economic viability assessments, and it can also be used to obtain statistical PV production data. The PVGIS provides sets of monthly averaged PV production daytime curves, sampled every $15 \mathrm{~min}$. Any of these evolution curves can be introduced as $P_{\mathrm{PVM}}$ into the QP in Fig. 2 after being adapted by the expected weather forecast. The resulting production model is relatively acceptable for clear days but lacks precision for overcast days when the actual PV production may differ greatly from the predicted model. This would mean that the PV + ES power plant would not be able to track the initial $P_{\text {ref }}^{*}$ due to the likelihood of saturations occurring in the ESS. The initial $P_{\text {ref }}^{*}$ is, therefore, a good statistical approach for long-term analyses, but not a precise approach for knowing what is really going to happen on a particular day.

Given that the current configuration of electricity markets worldwide is such that they always offer opportunities for generators to adjust their power commitment, some $P_{\text {ref }}^{*}$ refinements can be introduced during the daytime. In some of these markets, the power variations are traded on the so-called intraday electricity markets, whose frequency, duration, and number vary with the market. For the case of the Iberian market (Spain + Portugal), which has been taken as the framework for the analysis in this paper, it is divided into six sessions regularly distributed throughout the day.

Section III describes a methodology for taking advantage of the intraday markets, by adjusting the $P_{\text {ref }}^{*}$ in each of them to complete this EMS and to obtain a proper PV power commitment with minimum deviations and the corresponding cost penalties.

\section{ESTIMATION OF DAILY SOLAR ENERGY}

In order to overcome the limitations existing in the initial definition of the $P_{\text {ref }}^{*}$, which the plant committed itself to on the daily electricity market, some adjustments should be introduced as the day goes by to correct this power commitment on the intraday market. These adjustments basically consist of using the QP optimization, (5)-(9), to recalculate the $P_{\text {ref }}^{*}$ throughout the day using updated values of:

1) the current SOC of the ESS, whose deviation with regard to the one expected from the model allows deviations in the initially estimated PV plant operation to be corrected;

2) the PV energy production that the power plant will be able to provide on that particular day, which allows the $P_{\mathrm{PVM}}$ for the coming hours to be modified.

The current SOC is determined automatically from local measures. Conversely, some kind of mechanism has to be defined to obtain this updated approximation of the PV energy production expected for the day. A proposal is presented here.

\section{A. The Cloudiness Coefficient}

This proposal is based on the real-time calculation of the PV energy produced by the panels throughout the day $\left(E_{\mathrm{PV}}\right)$, and on comparing it with the expected ideally accumulated energy according to the PVGIS model ( $\left.E_{\mathrm{PVGIS}}\right)$ for clear-sky conditions. Both values of energy are calculated by integrating the corresponding power curves as time goes by, i.e., that provided by the PV panels $\left(P_{\mathrm{PV}}\right)$ and the standard power production profile statistically expected for that day according to PVGIS ( $\left.P_{\text {PVGIS }}\right)$, as can be appreciated in Fig. 4.

The quotient between these two energy values provides an instantaneous daily weather-dependent coefficient that varies in the daytime and which has been called the Cloudiness Coefficient $(C C)$. Thus, the $\mathrm{CC}$ is defined as

$$
\mathrm{CC}(t)=\frac{E_{\mathrm{PV}}(t)}{E_{\mathrm{PVGIS}}(t)} .
$$




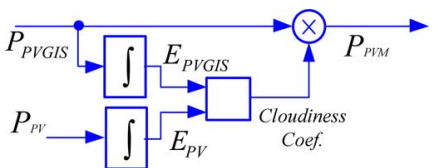

Fig. 4. Cloudiness coefficient-based $P_{\text {ref }}^{*}$ adjustment scheme.

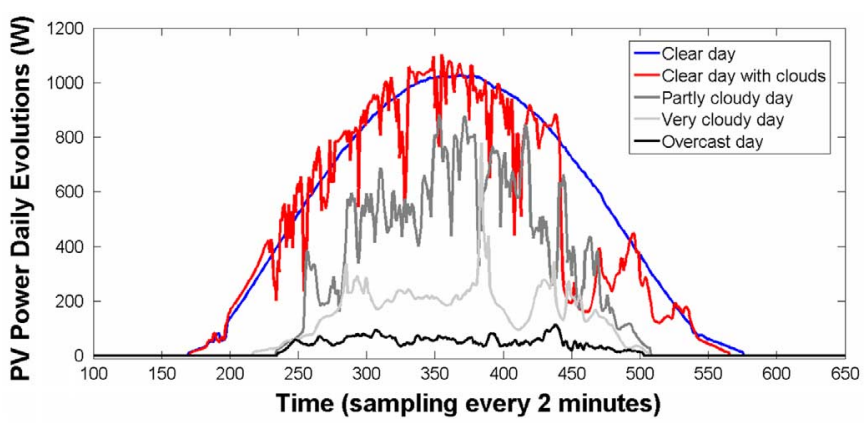

a)

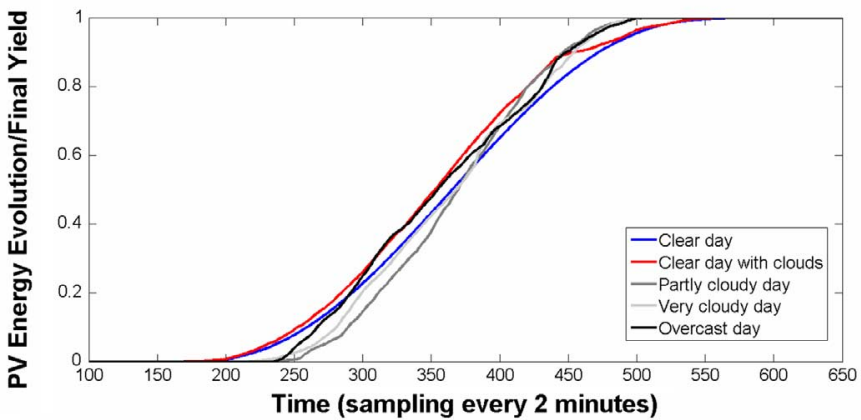

b)

Fig. 5. Evolutions throughout different weather-conditioned days of the (a) PV power production, and (b) PV energy related to the daily final energy yield.

This CC provides information on how cloudy each day has been up to the moment of the analysis, but it is also useful for estimating the energy yield which should be expected in the coming hours according to the following equations:

$$
\begin{aligned}
& E_{\text {estimated }}(t+k)=\frac{E_{\mathrm{PV}}(t)}{E_{\mathrm{PVGIS}}(t)} \cdot E_{\mathrm{PVGIS}}(t+k) \\
& E_{\text {estimated }}(t+k)=\mathrm{CC}(t) \cdot E_{\mathrm{PVGIS}}(t+k) .
\end{aligned}
$$

This estimation of the daily solar energy is performed by taking into account the fact that the PV energy yield in the daytime usually evolves in a regular way, regardless of the particular weather conditions associated to each day. This phenomenon is stated after analyzing real PV production data throughout one whole year. To obtain a random example, the power and energy evolutions during five days that register very different weather conditions (a clear day, a clear day with clouds, a partly cloudy day, a very cloudy day, and an overcast day) are introduced into Fig. 5 for a generic 1-kW PV power plant.

It can be observed in Fig. 5(a) how the five power curves are surrounded by a quadratic shape, clearly tied to the geometric relations between the relative positions of the Sun and the Earth's surface during the daylight hours, with more or less distortion depending on the cloud coverage. Conversely, the respective daily evolutions of their PV energy yield are more in-

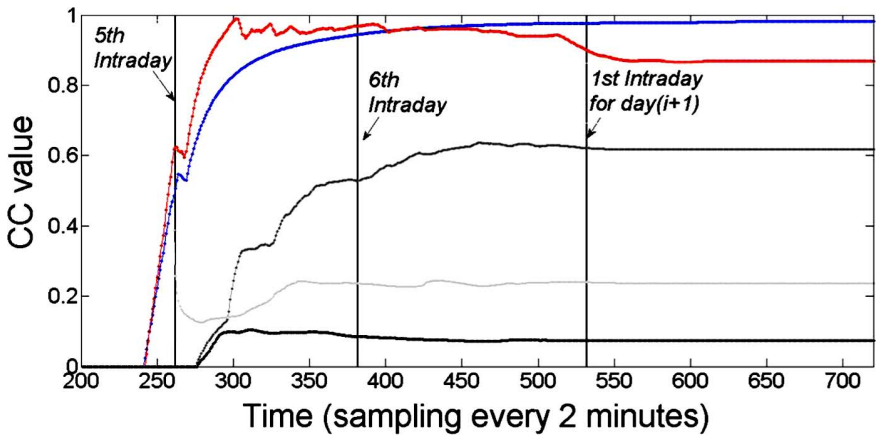

Fig. 6. Daily evolution of the $\mathrm{CC}$ value.

dependent from the instantaneous cloud coverage and follow a very cubic polynomial-like shape [Fig. 5(b)] which is derived from the integral of the corresponding PV power curves. It can be noted how these PV energy yield evolutions, represented in Fig. 5(b) as the progressive ratio (in pu) of the total PV energy produced by the power plant each day, are quite regular and, hence, they can be estimated in advance with a certain degree of accuracy.

According to these conclusions, by knowing $E_{\mathrm{PV}}$ and the PVGIS model, the PV energy yield in the coming hours can be estimated using (12). This estimation will reflect the same accuracy as that of the $\mathrm{CC}$ value at that moment, which makes it very important to use a value of $\mathrm{CC}$ as close as possible to the final one for each day. The PVGIS model will, therefore, be modified properly to provide an optimal $P_{\mathrm{PVM}}$, Fig. 4 .

An evolution of the value of the $\mathrm{CC}$ throughout each of the five days in Fig. 5 is represented in Fig. 6 (maintaining the same color code). In Fig. 6, it stands out how the $\mathrm{CC}$ value becomes more stable and closer to its final value as the day goes by, given that more and more actual PV production has been registered.

It is important then to analyze the daily evolution of the CC value throughout the year at the moment when $P_{\text {ref }}^{*}$ adjustments are accepted by the market. Note that out of the six intraday market sessions established in the Iberian electricity market, only three of them take place during the sunlight hours: the fifth, the sixth, and the first, corresponding to the next day's schedule (which still allows the power commitments for the day-on schedule to be modified [18]). The closure time for the three sessions, also highlighted in Fig. 6, are 8:45 A.M. (fifth), 12:45 P.M. (sixth), and 5:45 P.M. (first, day +1 ). To proceed with that analysis, Table I summarizes the mean value and the standard deviation of the error in the calculated value of the instantaneous $\mathrm{CC}$ with regard to the $\mathrm{CC}$ value at the end of the day

$$
\text { Error_CC }(t)=\frac{\mathrm{CC}(t)}{\mathrm{CC}(\text { final })} \text {. }
$$

Results in this table were obtained using actual irradiance values sampled every 2 min for the whole of the year 2009 . These provide an excellent idea of how accurate the calculation of $\mathrm{CC}$ is when finishing each of the bid-matching periods, which makes it possible to decide which intraday sessions can be used and how reliable the $\mathrm{CC}$ value is when refined $P_{\text {ref }}^{*}$ offers must be transmitted to the grid operator. 
TABLE I

Evolution of ANNUALly Averaged CC ERror

\begin{tabular}{|c|c|c|}
\hline Time & Mean value & Standard Deviation \\
\hline $8: 45 \mathrm{am}$ & 0.30 & 0.37 \\
\hline $12: 45 \mathrm{pm}$ & 0.93 & 0.24 \\
\hline $5: 45 \mathrm{pm}$ & 1.01 & 0.04 \\
\hline
\end{tabular}

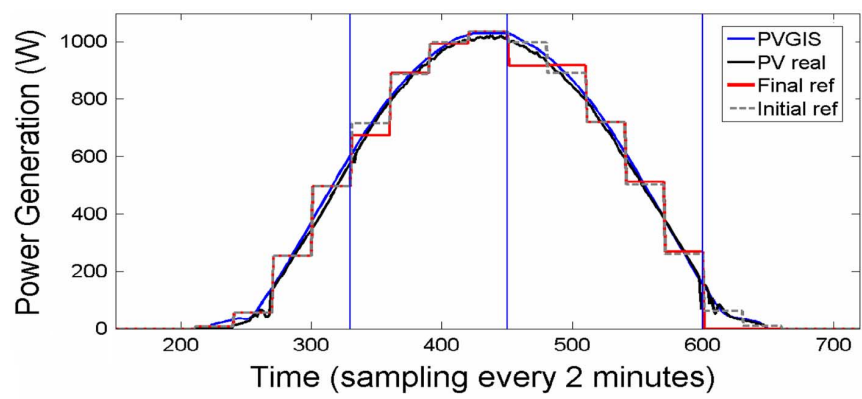

a)

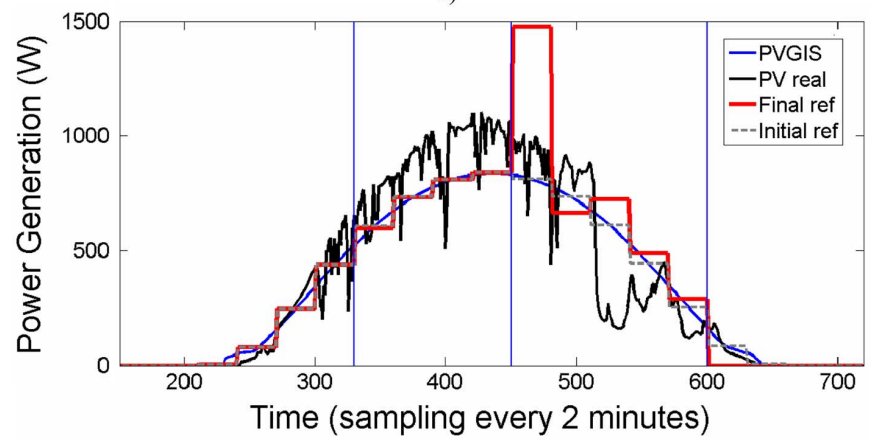

b)

Fig. 7. Refinements performed to $P_{\text {ref }}^{*}$ using the $\mathrm{CC}$ and taking advantage of the configuration of the intraday market for (a) a clear and (b) a cloudy day.

Thus, the mean error values in Table I show that, given that the calculation of the $\mathrm{CC}$ is based on the day-on-schedule real PV past production, many days (especially in winter) will not have registered enough PV production to establish a reliable daily solar energy estimation as early as 8:45 A.M.. Only during summer days will some information be useful. Therefore, the $\mathrm{CC}$ will only provide significant information and should be used to refine $P_{\text {ref }}^{*}$ in the sixth and the first intraday sessions.

To offset this potential lack of information in the morning hours, which can lead to quite erroneous operations during this period, updated meteorological information from the weather forecast service can be used in order to modify the $P_{\mathrm{PVM}}$ before the initial $P_{\text {ref }}^{*}$ calculations are performed. This option has also been considered and analyzed in Section IV.

\section{B. The Power Plant Reference Refinement}

According to the characteristics of the evolution of the CC and the profitability of the intraday sessions discussed for the Iberian electricity market, different functioning examples of the $P_{\text {ref }}^{*}$, generated with its corresponding adjustments, are introduced into Fig. 7 (for a clear and a cloudy day-(a) and (b), respectively) and in Fig. 8 (for a whole week).

For the cases represented in Fig. 7, the vertical lines define the time when the three intraday sessions start their operation periods (fifth at 11 P.M., sixth at 3 P.M., and first at 8 P.M.), which are the times when the $P_{\text {ref }}^{*}$ adjustments are finally applied. It

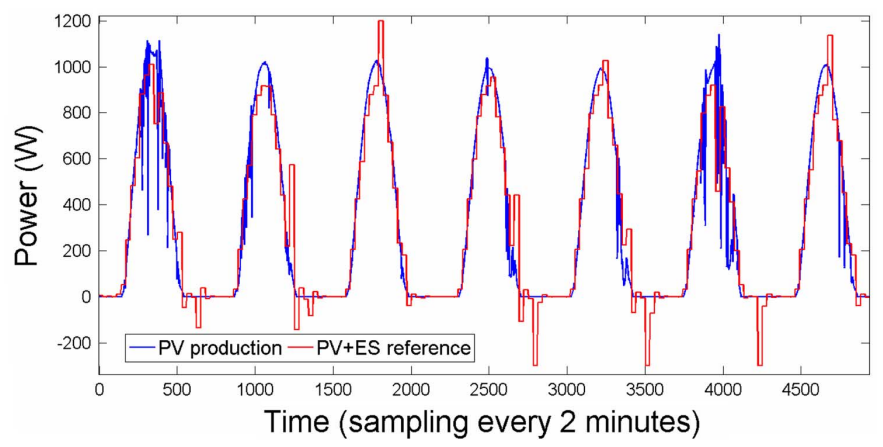

Fig. 8. Example of the hourly defined power reference for a week's operation of the PV + ES power plant with changing meteorological conditions.

stands out how, throughout the clear day in Fig. 7(a), the actual PV production almost completely overlaps the PVGIS-defined model for clear sky conditions. This validates the accuracy of the PVGIS database and the initial assumption of using its statistically modeled PV production as $P_{\mathrm{PVM}}$. Thus, it can be appreciated how the $P_{\text {ref }}^{*}$ refinements in the different intraday sessions are not significant (final ref versus initial ref) because the initial PV production forecast is precise and does not need important adjustments. Note how, during the sixth intraday session, the power reference values are slightly reduced in the first hour in order to compensate for the previously measured differences between the model and reality. The same adjustment is performed again during the first intraday session $($ day +1$)$.

Conversely, far more severe adjustments can be identified in Fig. 7(b) for the cloudy day. Since the actual PV production for that day differs from the PVGIS-predicted model quite a lot, and despite not being a critical day because good levels of production are still registered, the refined $P_{\text {ref }}^{*}$ (Final ref) significantly modify the initial production schedule that was calculated the day before (Initial ref). Therefore, while high production levels will be established when starting the sixth intraday period, no power will be established after the first $($ day +1$)$. In one case and the other, adjustments are made in order to keep the SOC level as close as possible to its reference value, in accordance with the QP optimization, trying to compensate for the deviations between the PVGIS-predicted model and the actual PV production experienced during the hours prior to each intraday period.

Finally, it stands out in Fig. 8 how the PV + ES reference $\left(P_{\text {ref }}^{*}\right)$ is adjusted in a different sense each day depending on the actual PV production registered during the morning hours. The adjustment is always performed in accordance with the target of keeping the ESS close to the $\mathrm{SOC}_{\text {ref }}$. Similarly, it can also be observed how the PV + ES plant will normally recharge during night hours to recover that $\mathrm{SOC}_{\mathrm{ref}}$. This will be done if deviations in the final SOC are registered. The recharge power capacity is limited in this analysis, as can also be appreciated.

Thus, with the introduction of the $\mathrm{CC}$ and the participation in the intraday market sessions, the PV power commitment can be refined throughout the day by correcting deviations between the forecasted and the actual daily PV production. This not only allows power deviation penalties to be avoided but also reduces the size of the ESS that is required. 
A comparison of the ESS energy capacity ratings required to operate the PV + ES plant in accordance with different configurations of the HCPS EMS is introduced in Section IV.

\section{ESS SIZING RESULTS}

A set of annually based simulations reproducing the operation of the PV + ES power plant, subject to four different EMS configurations, was performed. The goals of these simulations are:

1) to validate the effectiveness of the methodology for estimating the daily solar energy;

2) to determine the ESS energy capacity required for the proper operation of the power plant according to each of the four EMS configurations considered.

To do so, two sets of data were introduced into a Matlab simulation model which reproduces the PV power plant with ES. On the one hand, the first set corresponds to the real irradiance data registered in a Spanish location every 2 min throughout the whole of the year 2009. On the other hand, the second set contains the $P_{\text {ref }}^{*}$, this one being different depending on the EMS configuration. These are presented in the following to highlight the differences among configurations before introducing the corresponding variations in the ESS energy capacity needs.

\section{A. Generated Power References}

The four configurations which have been considered are:

- Type I-The basic HCPS EMS which generates a power reference with hourly adapted steps, exclusively calculated at the beginning of the day according to the PVGIS models without any further adjustment.

- Type II-This is also based on PVGIS models, but it recalculates $P_{\text {ref }}^{*}$ at every intraday market session to adjust it to the actual SOC (it takes into account deviations in the past daily energy production between the PVGIS model and the real PV production).

- Type III-Like the previous one, this configuration adjusts $P_{\text {ref }}^{*}$ at the intraday market sessions using the information about the actual SOC as feedback. However, this one also includes the $\mathrm{CC}$ for the sixth and the first intraday markets.

- Type IV-This last EMS configuration generates a $P_{\text {ref }}^{*}$ which combines the use of the $\mathrm{CC}$ in the sixth and the first intraday markets with an important weather correction in the PVGIS daily model to compensate for morning deviations in the production.

Therefore, the $P_{\text {ref }}^{*}$ will evolve in a quite different way throughout the day depending on the EMS configuration. This can be appreciated in Fig. 9(a). This figure shows how the first three configurations present a similar evolution in the morning hours (based on the PVGIS model) and start differing after the first intraday period. The adjustments in $P_{\text {ref }}^{*}$ can be easily understood with the complementary information provided by Fig. 9(b), which establishes the SOC evolutions of the ESS for that day as a function of the EMS. Note how the different adjustments help the control system to cope with its target of reducing the deviation of the SOC with regard to the $\mathrm{SOC}_{\mathrm{ref}}$.

As can be observed, the evolution of the SOC differs quite a lot from one strategy to the other. Therefore, depending on the

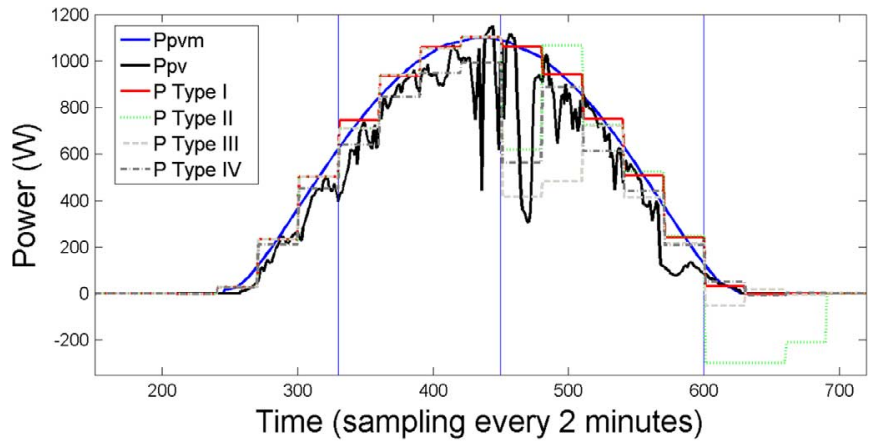

a)

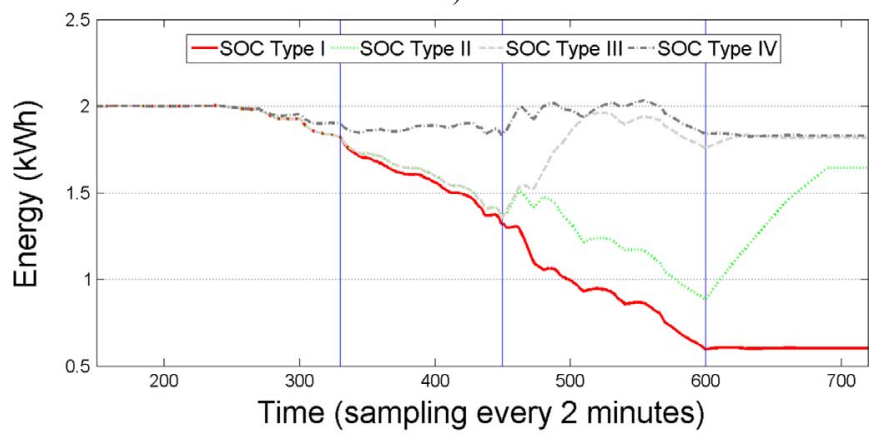

b)

Fig. 9. Results obtained as a function of the EMS configuration for a whole day operation: (a) references and (b) SOC evolution.

information considered to define the $P_{\mathrm{PVM}}$ and the number of times the QP optimization is launched, $P_{\text {ref }}^{*}$ will reflect the actual PV plant behavior to a greater or lesser degree by producing an SOC deviation that is more or less significant. This impacts the ESS energy capacity needs, which have been analyzed on an annual basis and are presented below.

\section{B. ESS Energy Capacity Ratings}

The two sets of data (actual PV power and $P_{\text {ref }}^{*}$ ) have been compared periodically throughout one whole year in 2-min periods (the sampling period of the actual irradiance curve). For each of these periods, the model calculates the amount of power to be exchanged by the ESS and how its SOC evolves considering that $\varepsilon_{c}=\varepsilon_{d}=0.9$, that the rated power of the ESS was set as large as required in order to prevent any $P_{\text {ref }}^{*}$ tracking errors in the PV + ES performance, and that the ESS power exchange in recharge was limited to $30 \%$ of the rated power of the PV plant. In this way, an SOC evolution analysis can be used to determine the number of periods in the year (percentage of time) that the ESS would saturate (being completely charged or discharged) when a certain energy capacity is supposed for the ESS.

Fig. 10 represents that percentage of time when the ESS, with a defined energy capacity, would not be able to guarantee the tracking of $P_{\text {ref }}^{*}$ due to saturations. Note that the energy capacity of the ESS has been introduced in per-unit values, taking the capacity factor $\left(C_{f}\right)$, the characteristic parameter of PV power plant associated to the region where they are installed, as the base value. This parameter establishes the annually averaged estimated daily PV energy production per installed $\mathrm{kW}$, and presents a value of $4.3 \mathrm{kWh} / \mathrm{kW}_{\text {peak }}$ in this case. This means 


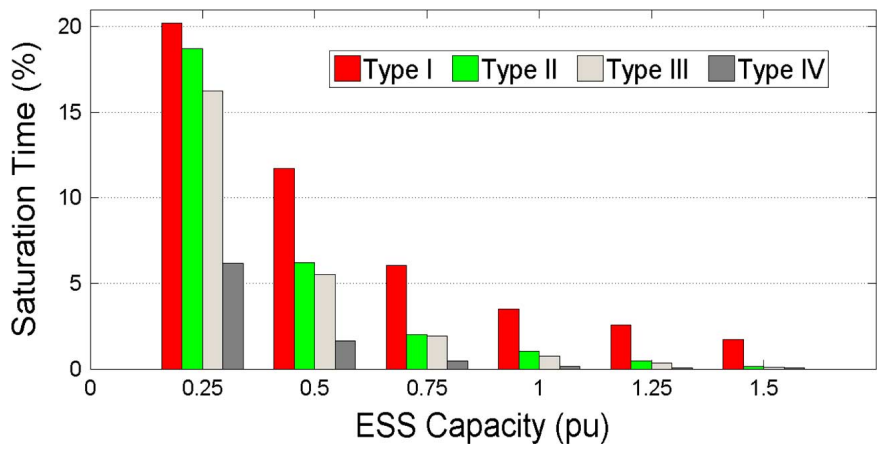

Fig. 10. Percentage of time in the year when the PV power plant cannot track the reference established for each of the four EMS configurations as a function of the ESS energy capacity.

that an ESS with an energy capacity of 0.25 pu corresponds to a $215-\mathrm{kWh}$ ESS in a $200-\mathrm{kW}$ PV plant.

Important differences in the percentage of time the plant is saturated with an equal ESS can be clearly observed among the four EMS configurations. Note that just by introducing the possibility of participating in the intraday market (Type II versus Type I), the percentage of saturated time is reduced by around $50 \%$ for ESS energy capacities over $0.5 \mathrm{pu}$. Type III provides better results thanks to the effectiveness of the CC, which allows saturations to be corrected in the afternoon and evening hours. Moreover, when adjustment actions based on weather forecasts are taken for the morning hours (Type IV) in order to avoid the initial uncertainty of the PVGIS statistical model, the ESS energy capacity needs are reduced even further. In fact, with the $\mathrm{CC}$ adjustment complemented by a morning weather-based correction, the saturation time with a 0.5 -pu ESS is reduced by a factor of 10 . Thus, the lowest storage capacity ratings to ensure proper performance of the PV + ES power plant are associated to this Type IV configuration. This requires an ESS with a capacity ranging from $25 \%$ to $50 \%$ of the PV power plant capacity factor (average daily energy production throughout the year). As an example, it is important to note how a 1-MW PV power plant could properly track the $P_{\text {ref }}^{*}$ defined by this EMS during $94 \%$ of the time throughout the year if an ESS with a capacity of 1.1 MWh was incorporated, compared to values of around 1.6 MWh for Types II and III or even $3 \mathrm{MWh}$ for the Type I configuration.

\section{Results Discussion}

It is important to conclude that a precise estimation of the ESS energy capacity ratings required to operate a PV + ES power plant under these EMS configurations can be extracted from this analysis, and that these results are statistically reliable given the one-year-long horizon which characterizes the analysis. Similar results can be obtained for the ESS power requirements to track the reference with a significant reliability ( $>98 \%$ throughout the year). In this regard, the ESS power ratings are found to be in the range of $50 \%-60 \%$ of the corresponding rated power of the PV power plant.

Thus, these results could be used by future PV power plant designers and promoters to analyze the economic viability of the extra investment represented by the introduction of an ESS, a higher cost that should be offset by increased benefits obtained by participating in the electricity pool. In this sense, regarding extra investment costs, these are far more heavily influenced by the energy capacity requirements than by the power requirements of the ESS. Thus, considering the levels of energy capacity required by the best control option (Type IV EMS configuration), the ESS would represent an extra economic investment of around $20 \%$ or $25 \%$ of the initial cost of the PV power plant. And this is obtained when considering prices for technologies such as $\mathrm{Li}$ ion batteries, which are expected to reduce their costs in the coming years. On the other hand, since the EMS proposed in this paper for PV power plants enables them to take part in the daily and intraday electricity markets, and given that the revenue obtained from the pool price complemented by the economic premium assigned to the PV production is more beneficial than the current feed-in tariffs in countries like Spain, the development of PV + ES power plants in accordance with the present control proposal in such a scenario could be economically viable in the short term.

\section{CONCLUSION}

This paper has analyzed a possible solution to enable PV power plants to enter the electricity markets while minimizing the extra costs derived from the introduction of an ESS.

The proposed solution is based on the definition of a new EMS for PV power plants with ES. This EMS, mainly focused on transforming the PV irradiance-dependent production into a constant-by-hours production, adopts various configurations. Thus, whereas the simplest one is only based on statistical data to establish the constant-by-hours power reference once a day, the others introduce different adjustments throughout the day in accordance with the updated information available at each moment. The four EMS configurations defined have been simulated and their performances compared. And then, the ESS ratings required to ensure proper tracking of the reference, defined by the control system, have been analyzed and quantified for each of them, the optimal configuration being the one which includes various weather corrections.

Hence, the analysis presented and the results obtained in this paper are a valuable and interesting reference that could be used by future PV plant promoters to obtain an approximation of the ESS ratings that their plants would require so as to be able to participate in the electricity markets with an increased reliability.

\section{REFERENCES}

[1] B. Bose, "Global warming: Energy, environmental pollution, and the impact of power electronics," IEEE Ind. Electron. Mag., vol. 4, no. 1, pp. 6-17, Mar. 2010.

[2] D. A. Halamay, T. K. A. Brekken, A. Simmons, and S. McArthur, "Reserve requirement impacts of large-scale integration of wind, solar, and ocean wave power generation," IEEE Trans. Sustain. Energy, vol. 2, no. 3, pp. 321-328, Jul. 2011.

[3] A. Nourai and D. Kearns, "Batteries included," IEEE Power Energy Mag., vol. 8, no. 2, pp. 49-54, Mar./Apr. 2010.

[4] S. Teleke, M. E. Baran, S. Bhattacharya, and A. Q. Huang, "Rule-based control of battery energy storage for dispatching intermittent renewable sources," IEEE Trans. Sustain. Energy, vol. 1, no. 3, pp. 117-124, Oct. 2010.

[5] P. Denholm and R. M. Margolis, "Evaluating the limits of solar photovoltaics (PV) in electric power systems utilizing energy storage and other enabling technologies," Energ Policy, vol. 35, pp. 4424-4433, Sep. 9, 2007. 
[6] D. Chattopadhyay, "Scale efficient network development to support renewable generation development," IEEE Trans. Sustain. Energy, vol. 2, no. 3, pp. 329-339, Jul. 2011.

[7] A. Jaeger-Waldau, European Comission Joint Research Centre Italy, Tech. Rep. JRC24807, PV status report 2011, Dec. 2011.

[8] A. Nourai, "Large-scale electricity storage technologies for energy management," in Proc. IEEE Power Eng. Soc. Summer Meeting, 2002, vol. 1 , pp. 310-315.

[9] S. Vazquez, S. M. Lukic, E. Galvan, L. G. Franquelo, and J. M. Carrasco, "Energy storage systems for transport and grid applications," IEEE Trans. Ind. Electron., vol. 57, no. 12, pp. 3881-3895, Dec. 2010.

[10] J. P. Barton and D. G. Infield, "Energy storage and its use with intermittent renewable energy," IEEE Trans. Energy Convers., vol. 19, no. 2, pp. 441-448, Jun. 2004.

[11] Y. Riffonneau, S. Bacha, F. Barruel, and S. Ploix, "Optimal power flow management for grid connected PV systems with batteries," IEEE Trans. Sustain. Energy, vol. 2, no. 3, pp. 309-320, Jul. 2011.

[12] M. Lafoz, L. Garcia-Tabares, and M. Blanco, "Energy management in solar photovoltaic plants based on ESS," in Proc. 13th EPE-PEMC Power Electronics and Motion Control Conf., 2008, pp. 2481-2486.

[13] EUROGIA + 2011 [Online]. Available: http://www.eurogia.com/

[14] PVGIS Database 2010 European Commission, Joint Research Centre [Online]. Available: http://re.jrc.ec.europa.eu/pvgis/index.htm

[15] H. Kanchev, D. Lu, F. Colas, V. Lazarov, and B. Francois, "Energy management and operational planning of a microgrid with a PV-based active generator for smart grid applications," IEEE Trans. Ind. Electron., vol. 58, no. 10, pp. 4583-4592, Oct. 2011.

[16] H. Fakham, D. Lu, and B. Francois, "Power control design of a battery charger in a hybrid active PV generator for load-following applications," IEEE Trans. Ind. Electron., vol. 58, no. 1, pp. 85-94, Jan. 2011.

[17] H. Beltran, E. Bilbao, E. Belenguer, I. Etxeberria-Otadui, and P. Rodriguez, "Evaluation of storage energy requirements for constant production in PV power plants," IEEE Trans. Ind. Electron. DOI: 10.1109/ TIE.2012.2202353

[18] A. Carbajo, "Los mercados eléctricos y los servicios de ajuste del sistema," Economía Industrial, vol. 364, pp. 55-62, 2007.

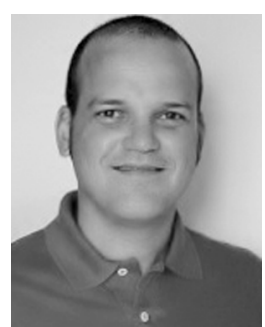

Hector Beltran received the M.Sc. degree in industrial engineering from the Universitat Jaume I (UJI), Castelló de la Plana, Spain, in 2004, and the Ph.D. degree in electrical engineering from the Technical University of Catalonia (UPC), Terrassa, Spain, in 2011.

During 2003, he worked at the European Centre for Nuclear Research (CERN), Geneva, Switzerland. From 2004 to 2006, he worked as a Researcher at the Electronic and Energy Departments of the Energy Technologycal Institute (ITE), València, Spain. Since 2006, he is an Assistant Professor of the Electrical Engineering Area at UJI. His current research interests include massive photovoltaic integration into the grid, energy-storage systems, and microgrids.

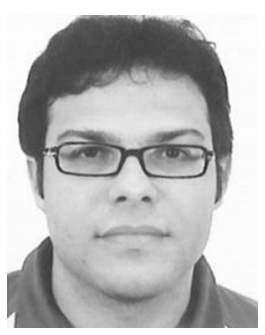

Emilio Pérez received the M.Sc. degree from the Universitat Jaume I (UJI), Spain, in 2002, and the Ph.D. degree from Universidad Politécnica de Valencia (UPV), Spain, in 2011.

Since 2006, he has been with the Industrial Systems Engineering and Design Department at UJI, where he currently works as a lecturer. $\mathrm{He}$ has published more than 15 conference papers and several international journal papers. His current research interests include model predictive control, convex optimization, and control of PV plants with

energy storage.

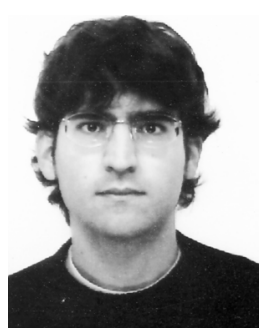

Néstor Aparicio (S'06-M'12) received the M.Sc degree from the Universitat Jaume I (UJI), Spain, in 2002, and the Ph.D. degree from Universidad Politécnica de Valencia (UPV), Spain, in 2011.

$\mathrm{He}$ is an Assistant Professor of the Electrical Engineering Area at UJI with research interests in the grid integration of wind-power generators. For six months, he visited the Institute of Energy Technology of Aalborg, Denmark and the Centre for Energy and Environmental Markets (CEEM), Sydney, Australia in 2006 and 2008, respectively.

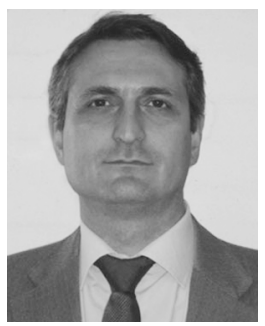

Pedro Rodriguez (S'99-M'04-SM'10) received the M.Sc. and Ph.D. degrees in electrical engineering from the Technical University of Catalonia (UPC), Barcelona, Spain, in 1994 and 2004, respectively.

He was a Postdoctoral Researcher at the Center for Power Electronics Systems, Virginia Tech, Blacksburg, in 2005, and in the Department of Energy Technology, Aalborg University (AAU), in 2006. He joined the faculty of UPC as an Assistant Professor in 1990, where he became the Director of the Research Center on Renewable Electrical Energy Systems in the Department of Electrical Engineering. He was also a Visiting Professor at the AAU from 2007 to 2011, acting as a co-supervisor of the Vestas Power Program. He still lectures Ph.D. courses at the AAU every year. Since 2011, he has been the Head of Electrical Engineering Division in Abengoa Research, Seville, Spain, although he is a part-time Professor at UPC. He has coauthored one book and more than 100 papers in technical journals and conference proceedings. He is the holder of seven licensed patents. His research interests include integration of distributed generation systems, smart grids, and design and control of power converters. 